\title{
DIMENSIONAL INTERPOLATION AND THE SELBERG INTEGRAL
}

\author{
V. GOLYSHEV, D. VAN STRATEN, AND D. ZAGIER
}

\begin{abstract}
We show that a version of dimensional interpolation for the Riemann-RochHirzebruch formalism in the case of a grassmannian leads to an expression for the Euler characteristic of line bundles in terms of a Selberg integral. We propose a way to interpolate higher Bessel equations, their wedge powers, and monodromies thereof to non-integer orders, and link the result with the dimensional interpolation of the RRH formalism in the spirit of the gamma conjectures.
\end{abstract}

The dimensions of spaces of sections of certain ample bundles on homogeneous spaces such as the grassmannian $G(k, N+k)$ of $k$-spaces in $\mathbb{C}^{N+k}$ can easily be interpolated as functions of the variable $N$ since they depend on $N$ polynomially. Deligne interpolated the spaces themselves to objects of a certain tensor category $\underline{\operatorname{Rep}}\left(G L_{t}\right)$, where $t$ should be thought of as $N+k$. For instance, beginning with the polynomial interpolation of $\chi_{\mathbb{P}^{N}}(\mathcal{O}(n))$ as $\left(\begin{array}{c}N+n \\ n\end{array}\right)=\left(\begin{array}{c}t-1+n \\ n\end{array}\right)$, one can take a step further and interpret $H\left(\mathbb{P}^{N}, \mathcal{O}(n)\right)$ as Sym $^{n} V_{t}^{*}$ in Deligne's category. We will leave $\mathbb{P}^{N}=G(1, N+1)=\mathbb{P}^{t-1}$ itself undefined, trying instead to operate with its vestiges in a consistent manner.

Different levels of interpolation appear naturally in this framework. For $t$ a natural number, the dimensions of actual objects in the usual category of representations $\operatorname{Rep}\left(G L_{t}\right)$ are given by the Weyl character formula and interpolate easily as functions of the highest weight. But how do the individual ingredients of Weyl's formula interpolate as functions of the length of the Dynkin diagram? Or, by Hirzebruch, the Euler characteristic of a vector bundle on a homogeneous space is the result of pairing up the Chern character with the Todd genus of the space; can both be interpolated naturally in such a way that the result of the pairing still behaves polynomially?

A level deeper, the Riemann-Roch-Hirzebruch numerology of projective spaces can be linked to the monodromy (or its version adapted to irregular connections) of the higher Bessel equations

$$
\left(D^{N+1}-z^{N+1}\right) \Psi(z)=0, \quad N \in \mathbb{N},
$$

where $D=z \frac{d}{d z}$. Does the interpolated 'differential equation'

$$
\left(D^{t}-z^{t}\right) \Psi(z)=0
$$

have rudiments of monodromy? Is this monodromy related to an interpolation of the RRH theorem?

Preliminary results show that the answers to these questions are positive. This short note should be viewed as a mere announcement of a more detailed version: we barely indicate the direction we are going by explaining a link between dimensional interpolation of the RRH formalism for line bundles on grassmannians and the Selberg integral (in 3 below) and showing how a version of the gamma conjecture for grassmannians might remain true (in 4 ).

We remark that several attempts to interpolate dimension as a continuous variable have been made in the past. Hausdorff defined fractional dimension $d$ in terms of the asymptotics of the number $\mathcal{N}(R)$ of balls of radius $R$ needed to cover $X$ :

$$
\mathcal{N}(R) \sim\left(\frac{1}{R}\right)^{d}
$$


J. von Neumann defined fractional dimensions in his Continuous Geometry in terms of projectors in operator algebras. In quantum field theory, dimensional regularization was introduced by t'Hooft and Veltman tHV72 and independently Bollini and Giambiagi BG72. The main observation is that the Feynman integral that belongs to a given Feynman diagram 'can be taken in any dimension $D$ ', basically because its integrand only contains (after Wick rotation) scalar products in euclidean space, [Eti99. For a review of various approaches to dimension, and specifically, a link with the theory of modular forms, we refer the reader to Man06.

Deligne's category, which we introduce in the next section, is not explicitly used anywhere in the paper. Nevertheless, its relevance should be clear from the context.

1. Deligne's category $\underline{\operatorname{Rep}}\left(G L_{t}\right)$. From the perspective of the Killing-Cartan-Weyl classification of simple Lie algebras and their representation theory in terms of highest weights, root systems, Weyl groups and associated combinatorics it is not so easy to understand the extreme uniformity in the representation theory that exists among different Lie groups. With possible application to a universal Chern-Simons type knot invariant in mind, P. Vogel Vog99 tried to define a universal Lie algebra, $\mathfrak{g}(\alpha: \beta: \gamma)$ depending on three Vogel parameters that determine a point $(\alpha: \beta: \gamma)$ in the Vogel plane, in which all simple Lie algebras find their place. The dimension of the Lie algebra $\mathfrak{g}(\alpha: \beta: \gamma)$ is given by a universal rational expression

$$
\operatorname{dim} \mathfrak{g}(\alpha: \beta: \gamma)=\frac{(\alpha-2 t)(\beta-2 t)(\gamma-2 t)}{\alpha \beta \gamma}, \quad t=\alpha+\beta+\gamma,
$$

and similar universal rational formulas can be given for the dimensions of irreducible constituents of $S^{2} \mathfrak{g}, S^{3} \mathfrak{g}$ and $S^{4} \mathfrak{g}$. Although the current status of Vogel's suggestions is unclear to us, these ideas have led to many interesting developments, such as the discovery of $E_{7 \frac{1}{2}}$ by Landsberg and Manivel, [LM02], LM04, [LM06b], LM06a], LM06a].

In order to interpolate within the classical $A, B, C, D$ series of Lie algebras, Deligne has defined $\otimes$-categories

$$
\underline{\operatorname{Rep}}\left(G L_{t}\right), \underline{\operatorname{Rep}}\left(O_{t}\right)
$$

where $t$ is a parameter that can take on any complex value. (The category $\underline{\operatorname{Rep}}\left(S p_{2 t}\right)$ is usually not discussed as it can be expressed easily in terms of the category $\underline{\operatorname{Rep}}\left(O_{T}\right)$ with $T=-2 t$.) If $n$ is an integer, there are natural surjective functors

$$
\underline{\operatorname{Rep}}\left(G L_{n}\right) \rightarrow \operatorname{Rep}\left(G L_{n}\right)
$$

In the tannakian setup one would attempt to reconstruct a group $G$ from its $\otimes$-category of representations $\operatorname{Rep}(G)$ using a fibre functor to the $\otimes$-category $V$ ect of vector spaces, but Deligne's category has no fibre functor and is not tannakian, or, in general, even abelian. (However, when $t$ is not an integer, the category is abelian semisimple.)

According to the axioms, in an arbitrary rigid $\otimes$-category $\mathcal{R}$ there exist a unit object $\mathbf{1}$ and canonical evaluation and coevaluation morphisms

$$
\epsilon: V \otimes V^{*} \rightarrow \mathbf{1}, \quad \delta: \mathbf{1} \rightarrow V \otimes V^{*}
$$

so that we can assign to any object a dimension by setting

$$
\operatorname{dim} V=\epsilon \circ \delta \in \operatorname{End}(\mathbf{1}) \in \mathbb{C} .
$$

A simple diagrammatic description of $\underline{\operatorname{Rep}}\left(G L_{t}\right)$ can be found in [CW12]. One first constructs a skeletal category $\underline{\operatorname{Rep}}_{0}\left(G L_{t}\right)$, whose objects are words in the alphabet $\{\bullet, \circ\}$. The letter • corresponds to the fundamental representation $V$ of $G L_{t}$, 。 to its dual $V^{*}$. $\mathrm{A} \otimes$-structure is induced by concatenation of words. The space of morphisms between two such words is the $\mathbb{C}$-span of a set of admissible graphs, with vertices the circles and dots of the two words. Such an admissible graph consists of edges between the letters of the 
two words. Each letter is contained in one edge. Such an edge connects different letters of the same word or the same letter if the words are different.

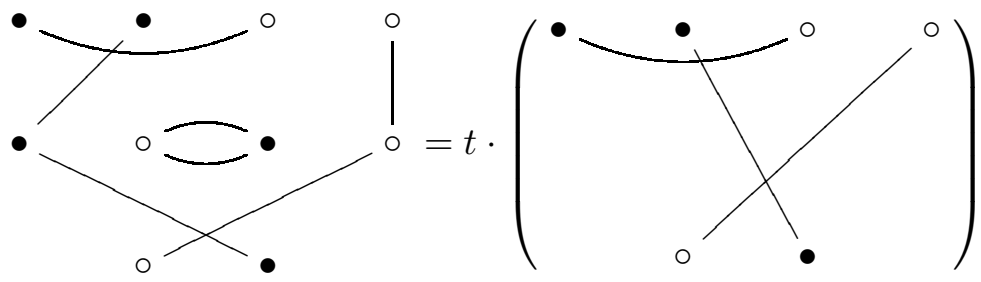

The composition is juxtaposition of the two graphs, followed by the elimination of loops, which results in a factor $t$.

Deligne's category is now obtained by first forming its additive hull by introducing formally direct sums and then passing to the Karoubian hull, i.e. forming a category of pairs $(W, e)$, consisting of an object together with an idempotent:

$$
\underline{\operatorname{Rep}}\left(G L_{t}\right)=\left(\underline{\operatorname{Rep}}_{0}\left(G L_{t}\right)^{\text {add }}\right)^{\text {Karoubi }} .
$$

Example. Consider the word $\bullet \bullet$ and the morphisms Id and Swap with the obvious meaning. One then can put

$$
S^{2} V=(\bullet \bullet, s), \quad \wedge^{2} V=(\bullet \bullet, a),
$$

where

$$
s=\frac{1}{2}(\mathrm{Id}+\mathrm{Swap}), \quad a=\frac{1}{2}(\mathrm{Id}-\mathrm{Swap})
$$

so that in $\underline{\operatorname{Rep}}\left(G l_{t}\right)$ one has:

$$
V \otimes V=(\bullet \bullet, I d)=S^{2} V \oplus \wedge^{2} V
$$

which upon taking dimensions is the identity

$$
t^{2}=\frac{t(t+1)}{2}+\frac{t(t-1)}{2}
$$

2. 'Spaces of sections' as objects in Deligne's category and the beta integral. As above, we assume that $n$ is a natural number. Write $t=N+1$ and let $V_{t}=V$ be the fundamental object of $\underline{\operatorname{Rep}}\left(G L_{t}\right)$ so that $\operatorname{dim} V_{t}=t$. We do not define the projective space $\mathbb{P}=\mathbb{P}^{N}$, but we can pretend that, in the sense of Deligne, the space of global sections is

$$
H\left(\mathcal{O}_{\mathbb{P}}(n)\right):=\operatorname{Sym}^{n}\left(V_{t}^{*}\right) \in \underline{\operatorname{Rep}}\left(G L_{t}\right) .
$$

Its dimension is then, as expected

$$
\chi\left(\mathcal{O}_{\mathbb{P}}(n)\right):=\operatorname{dim} H\left(\mathcal{O}_{\mathbb{P}}(n)\right)=\left(\begin{array}{c}
N+n \\
n
\end{array}\right),
$$

(interpreted in the obvious way as a polynomial in $N$ if $N \notin \mathbb{Z}$ ), so that e.g.

$$
\chi\left(\mathcal{O}_{\mathbb{P}^{1 / 2}}(2)\right)=\frac{3}{8} .
$$

The Poincaré series

$$
P(y):=\sum_{n=0}^{\infty} \chi\left(\mathcal{O}_{\mathbb{P}}(n)\right) y^{n}=\frac{1}{(1-y)^{N+1}},
$$

which is consistent with the idea that $\operatorname{dim} V_{t}=N+1$.

Returning to the question posed at the beginning, "is there a way to extend the interpolation of $\chi$ individually to the Chern and the Todd ingredients?', we reason as follows. If $X$ is a smooth projective $n$-dimensional variety, and $E$ a vector bundle on $X$, then the Euler characteristic

$$
\chi(X, E):=\sum_{i=0}^{n}(-1)^{i} \operatorname{dim} H^{i}(X, E)
$$


can be expressed in terms of characteristic numbers

$$
\chi(X, E)=\int_{X} \operatorname{ch}(E) \cdot \operatorname{td}(X) .
$$

Here the integral in the right hand side is usually interpreted as resulting from evaluating the cap product with the fundamental class $[X]$ on the cohomology algebra $H^{*}(X)$, and the Chern character and Todd class are defined in terms of the Chern roots $x_{i}$ of $E$ and $y_{i}$ of $T X$ :

$$
\operatorname{ch}(E)=\sum_{i=1}^{r} e^{x_{i}}, \quad \operatorname{td}(X)=\prod_{i=1}^{n} \frac{y_{i}}{1-e^{-y_{i}}} .
$$

The cohomology ring of an $n$-dimensional projective space is a truncated polynomial ring:

$$
H^{*}\left(\mathbb{P}^{N}\right)=\mathbb{Z}[\xi] /\left(\xi^{N+1}\right), \quad \xi=c_{1}(\mathcal{O}(1)),
$$

and it is not directly clear how to make sense of this if $N$ is not an integer. Our tactic will be to drop the relation

$$
\xi^{N+1}=0
$$

altogether, thinking instead of $\mathbb{Z}[\xi]$ as a Verma module over the $s l_{2}$ of the Lefschetz theory, and replacing taking the cap product with integration. As we will be integrating meromorphic functions in $\xi$, the polynomial ring is too small, and we put

$$
\hat{H}(\mathbb{P}):=\mathbb{Z}[[s]] \supset \mathbb{Z}[s] .
$$

One has

$$
\chi(\mathcal{O}(n))=e^{n \xi}, \quad \operatorname{td}(\mathbb{P})=\left(\frac{\xi}{1-e^{-\xi}}\right)^{N+1},
$$

so Hirzebruch-Riemann-Roch reads

$$
\chi(\mathcal{O}(n))=\left[e^{n \xi}\left(\frac{\xi}{1-e^{-\xi}}\right)^{N+1}\right]_{N}
$$

where $[\ldots]_{N}$ is the coefficient at $\xi^{N}$ in a series. This can be expressed analytically as a residue integral along a small circle around the origin:

$$
\chi(\mathcal{O}(n))=\frac{1}{2 \pi i} \oint e^{n \xi}\left(\frac{\xi}{1-e^{-\xi}}\right)^{N+1} \frac{d \xi}{\xi^{N+1}} .
$$

As it stands, it cannot be extended to non-integer $N$ since the factor $\left(1-e^{-\xi}\right)^{-N-1}$ is not univalued on the circle. The usual way to adapt it is to consider, for $n \geq 0$, the integral along the path going from $-\infty-i \varepsilon$ to $-i \varepsilon$, making a half-turn round the origin and going back, and choosing the standard branch of the logarithm. Because of the change in the argument this integral is equal to

$$
J(N, n)=\frac{e^{2 \pi i(N+1)}-1}{2 \pi i} \int_{-\infty}^{0} \frac{e^{n \xi}}{\left(1-e^{-\xi}\right)^{N+1}} d \xi,
$$

or, after the substitution $s=e^{\xi}$,

$J(N, n)=\frac{e^{2 \pi i(N+1)}-1}{2 \pi i} \int_{0}^{1} s^{n-1}(1-1 / s)^{-N-1} d s=\frac{\sin \pi(N+1)}{\pi} \int_{0}^{1} s^{n+N}(1-s)^{-N-1} d s$.

Using Euler's formulas

$$
\begin{gathered}
\Gamma(x) \Gamma(1-x)=\frac{\pi}{\sin \pi x} \\
\int_{0}^{1} s^{\alpha-1}(1-s)^{\beta-1} d s=\frac{\Gamma(\alpha) \Gamma(\beta)}{\Gamma(\alpha+\beta)},
\end{gathered}
$$

and

$$
\frac{\Gamma(N+n+1)}{\Gamma(n+1) \Gamma(N+1)}=\left(\begin{array}{c}
N+n \\
n
\end{array}\right),
$$


we arrive at a version of RRH 'with integrals':

Proposition 1. Let $n \in \mathbb{N}$. Assume $\operatorname{Re} N<0, N \notin \mathbb{Z}$. Interpret the Euler characteristic of $\mathbb{P}^{N}$ via formula (1). Then

$$
\chi_{\mathbb{P}}(\varnothing(n))=\frac{e^{2 \pi i(N+1)}-1}{2 \pi i} \int_{-\infty}^{0} \frac{e^{n \xi}}{\left(1-e^{-\xi}\right)^{N+1}} d \xi .
$$

3. The grassmannian and the Selberg integral. For $\mathbb{P}^{N}$, we ended up with the beta function, a one-dimensional integral, as the cohomology ring is generated by a single class $\xi$. In the cases where the cohomology ring is generated by $k$ elements, for example the grassmannian $G(k, N+k)$, we would like to see a $k$-dimensional integral appear in a natural way. For $N \in \mathbb{N}$ the cohomology ring of the grassmannian $\mathbb{G}:=G(k, N+k)$ is given by

$$
H^{*}(G(k, N+k))=\mathbb{C}\left[s_{1}, s_{2}, \ldots, s_{k}\right] /\left(q_{N+1}, q_{N+2}, \ldots, q_{N+k}\right),
$$

where the $s_{i}$ are the Chern classes of the universal rank $k$ sub-bundle and $q_{i}=c_{i}(Q)$ are formally the Chern classes of the universal quotient bundle $Q$ (so that the generating series of $q$ 's is inverse to that of $s$ 's). In the same vein as before, we set:

$$
\hat{H}^{*}(\mathbb{G}):=\mathbb{C}\left[\left[s_{1}, s_{2}, \ldots, s_{k}\right]\right]=\mathbb{C}\left[\left[x_{1}, x_{2}, \ldots, x_{k}\right]\right]^{S_{k}}
$$

by dropping the relations. A $\mathbb{C}$-basis of this ring given by the Schur polynomials

$$
\sigma_{\lambda}:=\frac{\operatorname{det}\left(x_{i}^{\lambda_{j}+k-j}\right)}{\operatorname{det}\left(x_{i}^{k-j}\right)}
$$

where $\lambda=\left(\lambda_{1}, \lambda_{2}, \ldots, \lambda_{k}\right)$ is an arbitrary Young diagram with at most $k$ rows. There is a Satake-type map for the extended cohomology:

$$
\text { Sat : } \hat{H}(\mathbb{G}) \rightarrow \wedge^{k} \hat{H}(\mathbb{P})
$$

obtained from the Young diagram by 'wedging its rows':

$$
\sigma_{\lambda} \mapsto s^{\lambda_{1}+k-1} \wedge s^{\lambda_{2}+k-2} \wedge \ldots \wedge s^{\lambda_{k}-1} .
$$

We are therefore seeking an expression for the values of the Hilbert polynomial of $G(k, N)$ in terms of a $k$-dimensional integral of the beta type involving $k$-wedging.

Euler's beta integral (3) has several generalizations. Selberg introduced [Sel44] an integral [FW08] over the $k$-dimensional cube

$S(\alpha, \beta, \gamma, k):=\int_{0}^{1} \ldots \int_{0}^{1}\left(s_{1} s_{2} \ldots s_{k}\right)^{\alpha-1}\left(\left(1-s_{1}\right)\left(1-s_{2}\right) \ldots\left(1-s_{k}\right)\right)^{\beta-1} \Delta(s)^{2 \gamma} d s_{1} d s_{2} \ldots d s_{k}$

where

$$
\Delta(s)=\Delta\left(s_{1}, s_{2}, \ldots, s_{k}\right)=\prod_{i<j}\left(s_{i}-s_{j}\right)
$$

and showed that it admits meromorphic continuation, which we will also denote by $S$.

Proposition 2. For $k \in \mathbb{N}, n \in \mathbb{Z}_{+}$, let $\chi\left(\mathcal{O}_{\mathbb{G}}(n)\right)$ denote the result of interpolating the polynomial function $\chi\left(\mathcal{O}_{G(k, k+N)}(n)\right)$ of the argument $N \in \mathbb{N}$ to $\mathbb{C}$. One has

$$
\chi\left(\mathcal{O}_{\mathbb{G}}(n)\right)=\frac{(-1)^{k(k-1) / 2}}{k !}\left(\frac{\sin \pi(N+1)}{\pi}\right)^{k} S(n+N+1,-N-k+1 ; 1, k) .
$$

Proof. The shortest (but not the most transparent) way to see this is to use the expressions for the LHS and the RHS in terms of the product of gamma factors found by Littlewood and Selberg respectively. By Selberg,

$$
S(\alpha, \beta, \gamma, k)=\prod_{i=0}^{k-1} \frac{\Gamma(\alpha+i \gamma) \Gamma(\beta+i \gamma) \Gamma(1+(i+1) \gamma)}{\Gamma(\alpha+\beta+(k+i-1) \gamma) \Gamma(1+\gamma)}
$$


By Littlewood [Lit42], for $N \in \mathbb{Z}_{>0}$ one has

$$
\chi\left(\mathcal{O}_{G(k, k+N)}(n)\right)=\frac{\left(\begin{array}{c}
N+n \\
n
\end{array}\right)\left(\begin{array}{c}
N+n+1 \\
n+1
\end{array}\right) \ldots\left(\begin{array}{c}
N+n+(k-1) \\
n+(k-1)
\end{array}\right)}{\left(\begin{array}{c}
N \\
0
\end{array}\right)\left(\begin{array}{c}
N+1 \\
1
\end{array}\right) \ldots\left(\begin{array}{c}
N+(k-1) \\
(k-1)
\end{array}\right)},
$$

where there are $k$ factors at the top and the bottom. Rearranging the terms in the RHS of (5) and using (2), we bring the $\Gamma$-factors that involve $\beta$ to the denominator in order to form the binomial coefficients at the expense of the sine factor.

As an example, for $k=2$ and $N=-1 / 2$, we get the Hilbert series

$$
\sum_{k=0}^{\infty} \chi\left(\mathcal{O}_{G(2,3 / 2)}(n)\right) y^{n}=1+6 \frac{t}{16}+60\left(\frac{t}{16}\right)^{2}+700\left(\frac{t}{16}\right)^{3}+8820\left(\frac{t}{16}\right)^{4}+\ldots
$$

which is no longer algebraic, but can be expressed in terms of elliptic functions.

More generally, one can consider a Selberg-type integral with an arbitrary symmetric function rather than the discriminant in the numerator and use separation of variables together with the Jacobi-Trudi formula in order to obtain similar expressions in terms of the gamma function in order to interpolate between the Euler characteristics of more general vector bundle on grassmannians (or the dimensions of highest weight representations of $\left.G L_{N+k}\right)$.

4. Towards a gamma conjecture in non-integral dimensions. The by now standard predictions of mirror symmetry relate the RRH formalism on a Fano variety $F$ to the monodromy of its regularized quantum differential equation. It is expected that this differential equation arises from the Gauss-Manin connection in the middle cohomology of level hypersurfaces of a regular function $f$ defined on some quasiprojective variety (typically a Laurent polynomial on $\mathbb{G}_{\mathrm{m}}^{d}$ ), called in this case a Landau-Ginzburg model of $F$. By stationary phase, the monodromy of the Gauss-Manin connection in a pencil translates into the asymptotic behavior of oscillatory integrals of the generic form $I(z)=\int \exp (i z f) d \mu\left(\mathbb{G}_{\mathrm{m}}^{d}\right)$, which satisfy the quantum differential equation of $F$, this time without the word 'regularized'. The asymptotics are given by Laplace integrals computed at the critical points, and the critical values of $f$ are the exponents occurring in the oscillatory integrals $I_{i}(z)$ that have 'pure' asymptotic behavior in sectors. One wants to express these pure asymptotics in terms of the Frobenius basis of solutions $\left\{\Psi_{i}(z)\right\}$ around $z=0$. The gamma conjecture GGI16 predicts that such an expression for the highest-growth asymptotic (arising from the critical value next to infinity) will give the 'gamma-half' of the Todd genus and therefore effectively encode the Hilbert polynomial of $F$ with respect to the anticanonical bundle. At first sight, none of this seems capable of surviving in non-integer dimensions. Yet, to return to the example of $G(2, N+2)$, define the numbers $c_{j}$ and $d_{j}$ by the expansions

$$
\begin{gathered}
\Gamma_{\mathbb{P}}^{(0)}(\varepsilon)=\Gamma(1+\varepsilon)^{N+2}=\sum_{j=0}^{\infty} d_{j} \varepsilon^{j}, \\
\Gamma_{\mathbb{P}}^{(1)}(\varepsilon)=\Gamma(1+\varepsilon)^{N+2} e^{2 \pi i \varepsilon}=\sum_{j=0}^{\infty} c_{j} \varepsilon^{j} .
\end{gathered}
$$

Put

$$
F(\varepsilon, z)=\sum_{k=0}^{\infty} \frac{z^{l+\varepsilon}}{\Gamma(1+l+\varepsilon)^{N+2}}
$$

and

$$
\Psi(\varepsilon, z)=\Gamma_{\mathbb{P}}(\varepsilon) F(\varepsilon, z)=\sum_{k=0}^{\infty} \Psi_{k}(z) \varepsilon^{k}
$$


Claim (rudimentary gamma conjecture). For fixed $N>2$ and $i, j$ in a box of at least some moderate size, one should have

$$
\lim _{z \rightarrow-\infty} \frac{\Psi_{i}(z) \Psi_{j}^{\prime}(z)-\Psi_{j}(z) \Psi_{i}^{\prime}(z)}{\Psi_{1}(z) \Psi_{0}^{\prime}(z)-\Psi_{0}(z) \Psi_{1}^{\prime}(z)}=\frac{c_{i} d_{j}-c_{j} d_{i}}{c_{1} d_{0}-c_{0} d_{1}}
$$

The LHS and RHS mimic, in the setup of formula (4), the $\sigma_{[j-1, i]}$-coefficients in the expansion of the 'principal asymptotic class' and the gamma class of the usual grassmannian: in the case when $N \in \mathbb{N}$ and $0 \leq i, j \leq N$ one would use the identification of 2-Wronskians of a fundamental matrix of solutions to a higher Bessel equation with homology classes of $G(2, N+2)$. Preliminary considerations together with numerical evidence suggest that the claim has a good chance to be true, as well as its versions for $G(k, N+k)$ with $k>2$.

The first-named author is grateful to Yuri Manin and Vasily Pestun for stimulating discussions. We thank Hartmut Monien for pointing us to [FW08.

\section{REFERENCES}

[BD16] Benjamin Braun and Robert Davis. Ehrhart series, unimodality, and integrally closed reflexive polytopes. Ann. Comb., 20(4):705-717, 2016.

[BG72] C. G. Bollini and J. J. Giambiagi. Dimensional renormalization : The number of dimensions as a regularizing parameter. Il Nuovo Cimento B (1971-1996), 12(1):20-26, Nov 1972.

[Bra13] Volker Braun. Counting points and Hilbert series in string theory. In Strings, gauge fields, and the geometry behind, pages 225-235. World Sci. Publ., Hackensack, NJ, 2013.

[BS13] Richard Beals and Jacek Szmigielski. Meijer G-functions: a gentle introduction. Notices Amer. Math. Soc., 60(7):866-872, 2013.

[CW12] Jonathan Comes and Benjamin Wilson. Deligne's category $\operatorname{Rep}\left(G L_{\delta}\right)$ and representations of general linear supergroups. Represent. Theory, 16:568-609, $2 \overline{012}$.

[Del02] P. Deligne. Catégories tensorielles. Mosc. Math. J., 2(2):227-248, 2002. Dedicated to Yuri I. Manin on the occasion of his 65 th birthday.

[EGNO15] Pavel Etingof, Shlomo Gelaki, Dmitri Nikshych, and Victor Ostrik. Tensor categories, volume 205 of Mathematical Surveys and Monographs. American Mathematical Society, Providence, RI, 2015.

[Eti99] Pavel Etingof. Note on dimensional regularization. In Quantum fields and strings: a course for mathematicians, Vol. 1, 2 (Princeton, NJ, 1996/1997), pages 597-607. Amer. Math. Soc., Providence, RI, 1999.

[Eti14] Pavel Etingof. Representation theory in complex rank, I. Transform. Groups, 19(2):359-381, 2014.

[Eti16] Pavel Etingof. Representation theory in complex rank, II. Adv. Math., 300:473-504, 2016.

[FW08] Peter J. Forrester and S. Ole Warnaar. The importance of the Selberg integral. Bull. Amer. Math. Soc. (N.S.), 45(4):489-534, 2008.

[GGI16] Sergey Galkin, Vasily Golyshev, and Hiroshi Iritani. Gamma classes and quantum cohomology of Fano manifolds: gamma conjectures. Duke Math. J., 165(11):2005-2077, 2016.

[GM14] Vasily Golyshev and Anton Mellit. Gamma structures and Gauss's contiguity. J. Geom. Phys., 78:12-18, 2014.

[GW11] Benedict H. Gross and Nolan R. Wallach. On the Hilbert polynomials and Hilbert series of homogeneous projective varieties. In Arithmetic geometry and automorphic forms, volume 19 of Adv. Lect. Math. (ALM), pages 253-263. Int. Press, Somerville, MA, 2011.

[Lit42] D. E. Littlewood. On the number of terms in a simple algebraic form. Proc. Cambridge Philos. Soc., 38:394-396, 1942 .

[Lit43] D. E. Littlewood. On the number of terms in a simple algebraic form under the symplectic group. Proc. Cambridge Philos. Soc., 39:197-199, 1943.

[LM02] J. M. Landsberg and L. Manivel. Triality, exceptional Lie algebras and Deligne dimension formulas. Adv. Math., 171(1):59-85, 2002.

[LM04] J. M. Landsberg and L. Manivel. Series of Lie groups. Michigan Math. J., 52(2):453-479, 2004.

[LM06a] J. M. Landsberg and L. Manivel. The sextonions and $E_{7 \frac{1}{2}}$. Adv. Math., 201(1):143-179, 2006.

[LM06b] J. M. Landsberg and L. Manivel. A universal dimension formula for complex simple Lie algebras. Adv. Math., 201(2):379-407, 2006. 
[Man85] Yu. I. Manin. New dimensions in geometry. In Workshop Bonn 1984 (Bonn, 1984), volume 1111 of Lecture Notes in Math., pages 59-101. Springer, Berlin, 1985.

[Man06] Yuri I. Manin. The notion of dimension in geometry and algebra. Bull. Amer. Math. Soc. (N.S.), 43(2):139-161, 2006.

[MV17] A. Matsuo and A. P. Veselov. Universal formula for the Hilbert series of minimal nilpotent orbits. Proc. Amer. Math. Soc., 145(12):5123-5130, 2017.

[Opd99] Eric M. Opdam. Cuspidal hypergeometric functions. Methods Appl. Anal., 6(1):67-80, 1999. Dedicated to Richard A. Askey on the occasion of his 65th birthday, Part I.

[Sel44] Atle Selberg. Remarks on a multiple integral. Norsk Mat. Tidsskr., 26:71-78, 1944.

[tHV72] Gerard 't Hooft and M. J. G. Veltman. Regularization and Renormalization of Gauge Fields. Nucl. Phys., B44:189-213, 1972.

[Vog99] Pierre Vogel. The universal Lie algebra. Preprint, 1999.

Algebra And Number Theory Laboratory

INSTITUTE FOR INFORMATION TRANSMISSION PROBLEMS

Bolshoi Karetny 19, Moscow 127994, Russia

golyshev@mccme.ru

Institut FÜR MATHEMATIK

Johannes GutenberG-Universität

Staudingerweg 9, 4. OG 55128 Mainz, Germany

straten@mathematik.uni-mainz.de

Max Planck Institut FÜr Mathematik

Vivatsgasse 7, 53111 Bonn, Germany

and

International Centre for Theoretical Physics

Via Miramare, Trieste, Italy

dbz@mpim-bonn.mpg.de 